Relations industrielles

Industrial Relations

\title{
Une politique de l'apprentissage II
}

\section{Gérard Tremblay}

Volume 2, numéro 4, décembre 1946

URI : https://id.erudit.org/iderudit/1023831ar

DOI : https://doi.org/10.7202/1023831ar

Aller au sommaire du numéro

Éditeur(s)

Département des relations industrielles de l'Université Laval

ISSN

0034-379X (imprimé)

1703-8138 (numérique)

Découvrir la revue

Citer cet article

Tremblay, G. (1946). Une politique de l'apprentissage II. Relations industrielles / Industrial Relations, 2(4), 6-8. https://doi.org/10.7202/1023831ar

Tous droits réservés (C Département des relations industrielles de l’Université Laval, 1946
Ce document est protégé par la loi sur le droit d'auteur. L'utilisation des services d'Érudit (y compris la reproduction) est assujettie à sa politique d'utilisation que vous pouvez consulter en ligne.

https://apropos.erudit.org/fr/usagers/politique-dutilisation/ 


\section{UNE POLITIQUE DE L'APPRENTISSAGE}

(Suite de la page 8)

se faire sur le chantier avec toutes les difficultés que l'on devine telles que le manque d'intérêt du compagnon à développer les connaissances de l'apprenti, l'hésitation de l'employeur à engager un ouvrier qui ne produit pas, la négligence de l'apprenti à développer ses connaissances techniques et à prendre le goût du métier.

L'activité accrue dans l'industrie de la construction à la suite de la disparition des contrôles fédéraux sur les matériaux et en raison du manque d'habitations et d'édifices publics, a fait amèrement réaliser la rareté de la main-d'œuvre qualifiée dans l'industrie du bâtiment. Cette rareté de la main-d'œuvre a même provoqué une surenchère durant toute l'année 1946 malgré le blocage des salaires par l'arrêté fédéral C.P. 9384. Les entrepreneurs en bâtiment du district de Montréal de même que les organisations ouvrières affiliées aux unions internationales et aux syndicats catholiques avaient déjà exposé à l'honorable M. Barrette, ministre du Travail, les difficultés qu'ils prévoyaient. Cette intervention est même à la source de la Loi d'aide à l'apprentissage qui, on le sait, permet aux employeurs, aux organisations ouvrières et aux comités paritaires de former une Commission d'apprentissage dont le but est de former de nouveaux hommes de métier ou de compléter les connaissances techniques des compagnons.

La Commission d'apprentissage de Montréal s'est mise à l'œuvre dès l'an passé. Grâce à la bienveillance du ministre du Travail, elle a obtenu l'utilisation de l'ancienne Ecole d'avionnerie, de l'avenue Laurier est; elle a transformé les lieux en école spécialisés pour les principaux métiers du bâtiment : briqueteur, plâtrier, peintre, charpentier, ferblantier, plombier et poseur d'appareils de chauffage, électricien. Jusqu'à janvier 1946, 194 jeunes ouvriers se sont inscrits aux cours théoriques et pratiques, en spécialisant comme suit : briqueteurs, 71 ; plâtriers, 14 ; peintres, 23 ; charpentiers, 32 ; ferblantiers, 13 ; plombiers et poseurs d'appareils de chauffage 23 ; électriciens, 18 . Tous ont été placés sitôt leurs cours terminés à des taux oscillant autour de $\$ 0.70$ l'heure. Une enquête faite par la Commission, afin de connaître l'appréciation patronale de ces jeunes ouvriers, révéla que leurs employeurs en étaient très satisfaits, qu'ils en demandaient de nouveaux de façon à satisfaire aux besoins du marché du travail. Au 31 octobre 1946, 137 apprentis étaient inscrits ; ils termineront leurs cours de six mois au printemps et seront absorbés immédiatement à des conditions avantageuses. Pour juin 1947, le programme comporte la mise sur le marché de 189 apprentis, dont 50 briqueteurs. Pour de 500 ouvriers du bâtiment auront été ainsi formés au cours des années 1946 et 1947.

Nous avons eu l'avantage de visiter l'École et de nous rendre compte de l'excellence de l'enseignement théorique et pratique par les instructeurs et moniteurs recrutés parmi les contremaîtres et les ouvriers les plus compétents de Montréal. Le moral des apprentis est élevé parce qu'ils apprennent à maîtriser un métier et réalisent que, dans quelques mois, ils seront en mesure, qu'ils soient des vétérans ou des civils, de gagner leur vie honorablement tout en contribuant à la prospérité canadienne.

D'autres centres d'apprentissage du bâtiment se développent; celui de Chicoutimi sera en mesure de placer 43 apprentis, finissant au début de janvier 1947, et 55 au cours de l'été pour les métiers de briqueteur, plâtrier, peintre et charpentier.

Le centre d'apprentissage de Sherbrooke libérera au début de l'année prochaine 74 apprentis et 65 vers le milieu de 1947. Le centre d'apprentissage de Sherbrooke forme des briqueteurs, des peintres, des charpentiers, des plombiers et des électriciens.

Voilà des réalisations qu'il convenait de signaler.

Nous trouvons excellente la formule d'intéresser les corps professionnels à la formation professionnelle. C'est une responsabilité qui leur incombe de droit naturel. Le rôle de l'Etat doit plutôt être d'ordre supplétif. Au surplus, le ministère du Travail aide généreusement ces Commissions d'apprentissage à même ses crédits. A signaler également l'initiative heureuse prise par les Comités paritaires de chacun de ces centres qui ont su puiser dans leurs réserves administratives pour aider au financement des Commissions d'apprentissage ; on ne pouvait mieux utiliser l'argent provenant des contributions des employeurs et des ouvriers de l'industrie et des métiers intéressés.

Le Bulletin des Relations industrielles tenait à signaler ces deux intéressantes initiatives de l'industrie de la chaussure et de l'industrie du bâtiment. Ce bref exposé met en lumière deux formules de formation professionnelle, la première issue de la collaboration directe de l'Etat et des corps professionnels, la seconde émanant des corps professionnels eux-mêmes.

Gérard Tremblay.

\section{LA PAIX INDUSTRIELLE DANS LE QUEBEC}

(Suite de la page \&)

vie et en définitive, éviter la plus grande cause de conflits du travail? Est-ce que vraiment toutes les entreprises se soucient d'adapter leur politique à la mentalité d'une population qui est française et catholique d'esprit?

Il faut espérer qu'elles profiteront de cette situation privilégiée dont elles jouissent actuellement, non pas pour oublier les problèmes qui se posent, en retarder la solution et s'endormir dans une fausse quiétude, mais pour opérer les réformes nécessaires et conserver à notre population ouvrière la belle mentalité qu'elle possède, car on doit toujours avoir présent à l'esprit que le bon moral dans une entreprise est une chose facile à perdre, difficile à créer et qu'il faut sans cesse cultiver.

Gerard Dion.

\section{INDUSTRIAL PEACE IN QUEBEC}

(From page 2)

short, so that the greatest cause of labour disputes may be eliminated? Are all business or industrial concerns trying to adapt their policy to the mentality of a french and catholic-minded population?

It is to be hoped that they will take advantage of the privileged circumstances in which they are actually placed to bring about all necessary reforms and help to preserve our workers' fine mentality, for one must always bear in mind that goodwill, in any undertaking, is hard to create and easily destroyed.

Gerard Dion. 


\section{NOS PUBLICATIONS}

\section{RAPPORT DU PREMIER CONGRÈS DES RELATIONS INDUSTRIELLES DE LAVAL - (\$1.00)}

Le Rapport du premier Congrès des Relationd industrielles de Laval contient les textes des conférences prononcées au Château Frontenac, Québec, le 11 mars 1946, sur les sujets suivants :

a) L'Université et les relations industrielles - T. R. P. Georges-Henri Lévesque, o.P., doyen de la Faculté des Sciences sociales ;

b) L'Evolution des relations industrielles au Canada - M. GÉrard Tremblay, sous-ministre du Travail et directeur du Département des Relations industrielles de Laval ;

c) L'apprentissage - M. LÉonce Girard, secrétaire du Comité paritaire de l'Industrie de la chaussure de la province de Québec ;

d) Fatigue industrielle et productivité - Dr Lucien Brouna, chef du Département d'Hygiène et de Physiologie appliquée à la Faculté de Médecine de l'Úniversité Laval et directeur des Recherches médicales à l'Aluminum Co. of Canada, Limited ;

e) Conciliation, arbitrage et tribunaux du travail - Mtre Lovis-PhilipPe Pigeon, c.R., professeur à la Faculté de Droit et à la Faculté des Sciences sociales de Laval ;

f) Les relations industrielles dans l'Etat moderne - M. JEF Rens, sous-directeur du Bureau international du Travail.

\section{CAHIERS DU SERVICE EXTÉRIEUR D'ÉDUCATION SOCIALE $\$ 0.15$ l'unité}

Vol. II, $\mathrm{n}^{\circ}$ 4-Programme de recherches sociales pour le Québec- EvereTt C. HuGHEs.

n. 8- La juridiction du travail - HeNRI BINET.

Vol. III, $n^{\circ} 1$ - Le chômage dans l'après-guerre - MaU Rice Lamontagne.

n 5 - La Déclaration de Philadelphie - Jean-Pierre Després.

$\mathrm{n}^{\circ} 8$ - Le marché du travail et les unions ouvrières - Je an-PiERre Després.

n० 9 - Nécessité d'une évolution du Droit civil - Mtre Louis-Ph. Pigeon.

Vol. IV, no 2 - Sécurité syndicale et Convention collective - Gérard Dion.

On peut se procurer ces publications en s'adressant au Département des Relations industrielles, Faculté des Sciences sociales, 2, rue de l'Université, Québec.

\section{*MA CONVERSION AU SYNDICALISME CATHOLIQUE» (1)}

Débordés par les exigences de l'action, les chefs syndicaux du Canada français ont rarement de loisirs pour publier des ouvrages dans ce domaine si important. Aussi, est-ce avec plaisir que l'on salue $M a$ conversion au syndicalisme catholique de M. Alfred Charpentier, ancien président général de la Confédération des Travailleurs catholiques du Canada.

Dans cet exposé qui, au premier abord, semble tout à fait personnel, l'auteur est incidemment amené à décrire des tendances qui existaient à cette époque (1907-1918) au sein des groupements ouvriers du Québec. Il laisse, en outre, percevoir le dur combat qui s'est livré en l'âme des chefs ouvriers pour se dégager de l'internationalisme neutre et diriger leur action d'après les principes de la doctrine de l'Église et les exigences de notre milieu. Leurs difficultés naissaient de leur attachement légitime à une forme de syndicalisme à laquelle ils s'étaient donnés en toute loyauté et dans le fait de voir, ches nous, des prêtres se mêler de problèmes auxquels ils étaient, jusque là, demeurés étrangers.

Le lecteur est mis en contact avec des figures sympathiques comme celles du R. P. Archambault, de l'abbé Maxime Fortin et de M. Arthur Marois, qui, chacun à leur façon, contribuèrent à former le futur président général de la C.T.C.C. On constate, aussi, l'influence jouée, chez nous, dans la naissance et l'expansion du syndicalisme catholique et national par l'œuvre des retraites fermées, l'A.C.J.C., l'École sociale populaire et le journalisme catholique.
Dans son volume, l'auteur ne fait, en somme, que présenter des extraits de conférences qu'il avait prononcées, ici et là, pendant qu'il était membre actif de l'internationale ou à ses débuts dans le syndicalisme catholique et des échanges de correspondance avec les journaux et, surtout, avec son ami, M. Marois. Par souci d'exactitute, l'auteur s'est peut-être trop confiné à reproduire des textes déjà composés, ce qui ne laisse de nuire un peu à la clarté de son récit.

Le livre de M. Charpentier est intéressant pour tous ceux qui ont à travailler dans le domaine social et particulièrement pour ceux qui sont trop jeunes pour avoir assisté à la naissance du mouvement syndical catholique. Il leur permettra de comprendre que dans toute action, il faut tenir compte des faits, que la prudence doit se joindre au zèle, que l'intransigeance dans des questions où il peut y avoir du plus et du moins n'est pas toujours le meilleur moyen de gagner les autres à sa cause. Il est assez intéressant de comparer les tendances actuelles du mouvement ouvrier chrétien avec ce que l'on en pensait dès le début.

Ardent, convaincu, travailleur acharné, l'auteur possède aujourd'hui une expérience sans pareille. Nous espérons qu'il continuera d'écrire les différentes phases de l'histoire de la C.T.C.C. et nous aimerions aussi lire sur le même sujet les souvenirs du pionnier de ce mouvement, M. l'abbé Maxime Fortin.

\section{Gérard Dion.}

(1) Volume de 240 pages, Editions Fides, 1946. 


\section{UNE POLITIQUE DE L'APPRENTISSAGE}

Nous avons exposé dans un article intitulé : Une Politique de l'Apprentissage, publié dans le numéro d'octobre de ce Bulletin, les conditions du développement rationnel de l'apprentissage. La cinquième condition énoncée était : Collaboration de l'État et des corps professionnels à tous les stades de l'enseignement technique et dans tous ses secteurs. Cette collaboration ne peut se faire que par le concours de comités consultatifs formés dans chaque métier ou dans chaque industrie qui aident à la préparation des programmes, à l'orientation des élèves et même à leur placement dans l'industrie. La Loi d'aide à l'apprentissage, votée l'an dernier par l'Assemblée législative, avait comme fin principale de réaliser et pour le moins de rendre possible cette collaboration.

Nous examinerons les initiatives prises par deux Commission d'apprentissage depuis leur formation : celle de l'industrie de la chaussure de Montréal et celle des métiers du bâtiment du même endroit.

La Commission d'apprentissage de l'industrie de la chaussure avait été précédée par un organisme désigné sous le nom de " l'Institut de la chaussure ". Le 23 août 1945, par l'arrêté en conseil $n^{\circ} 3431$, la Commission d'apprentissage succédait à cet organisme. Les buts de l'Institut de la chaussure comme ceux de la Commission d'apprentissage sont les suivants :

$1^{\circ}$ Rappeler aux employés les connaissances acquises à l'école et leur en montrer l'application pratique dans l'industrie. Faire le lien entre l'école et l'activité industrielle ;

$\mathscr{Z}^{\circ}$ Donner à l'employé la fierté de son métier en lui faisant connaître l'industrie, sa valeur économique, son histoire et les lois qui la régissent ;

$\boldsymbol{s}^{\circ}$ Donner, par le fait même, à l'industrie une maind'cuvre plus stable, qui cherche non seulement à vivre de l'industrie, mais à l'améliorer ;

$4^{\circ}$ Donner a l'employé des connaissances générales pour lui permettre de progresser dans son métier et d'occuper des fonctions plus élevées ;

$5^{\circ}$ Donner, par le fait même, à l'industrie des contremantres et des surintendants ;

$6^{\circ}$ Faire prendre à l'industrie la responsabilité de la préparation efficace de ses ouvriers par la mise en valeur d'un système complet de formation professionnelle ;

$y^{\circ}$ Suivre l'exemple des autres industries et des autres pays qui ont établi un lien entre l'école et le travail et en ont retiré de grands avantages ;

$8^{\circ}$ Conserver l'industrie dans la province de Québec en lui donnant les moyen de concurrencer avec les autres provinces et les autres pays.

On en conviendra, ces buts se rapprochent considérablement de ceux de l'enseignement spécialisé officiel. La seule différence est que l'enseignement spécialisé dans les conditions actuelles se développe sous l'initiative de l'Etat alors que la Commission d'apprentissage déploie ses activités sous l'initiative des corps professionnels.
Il est essentiel de faire converger ces deux forces vers une même fin, de les faire coopérer dans la meilleure harmonie afin que, de part et d'autre, elles donnent leur plein rendement dans l'obtention des buts à atteindre. La Commission d'apprentissage de l'Industrie de la chaussure l'a compris car elle a offert son entière coopération à l'enseignement spécialisé qui l'a, du reste, agréée volontiers. Si bien qu'aujourd'hui, nous constatons que l'École centrale des Arts et Métiers a ouvert dans l'ancien immeuble de l'Université de Montréal, rue SaintDenis, une école professionnelle de la chaussure à l'établissement de laquelle participe la Commission d'apprentissage.

Avant d'en arriver à ce stage de coopération, l'Institut de la chaussure avait donné, en 1945, 1,484 heures de cours à des groupes spécialisés d'auditeurs de 10 à 15 personnes. 969 ouvriers, répartis en 77 groupes, ont bénéficié des cours et 748 d'entre eux ont obtenu des certificats au 1er novembre 1945. Au 31 mars 1946, 87 groupes comprenant 1,482 auditeurs avaient reçu 29,877 heures de cours. Des certificats ont été remis à 1,417 élèves.

La Commission d'apprentissage de l'Industrie de la chaussure, comme je l'ai dit, collabore maintenant avec l'enseignement spécialisé sous la direction de l'honorable monsieur Sauvé, ministre du Bien-Etre social et de la Jeunesse. Une école professionnelle de la chaussure s'organise ; elle formera des ouvriers qualifiés pour tous les métiers de la chaussure : tailleurs, patronistes, ouvriers du cuir à semelles, couseurs et finisseurs. On comprendra l'importance de l'initiative si on tient compte que la province de Québec compte, à l'heure actuelle, 184 manufactures de chaussures employant plus de 14,000 ouvriers qui ont à leur crédit les deux-tiers de la production de la chaussure du Ganada.

Les cours techniques qui seront donnés à l'Ecole centrale des Arts et Métiers pourront également l'être dans les autres centres de production de la chaussure tels que Québec, Trois-Rivières, Saint-Hyacinthe, Plessisville, etc. La Commission d'apprentissage coopère avec l'École, aide au recrutement de ses instructeurs et moniteurs, prépare les programmes et les adapte aux besoins de l'industrie. Nous avons l'assurance que cette collaboration aidera puissamment au développement de l'industrie et permettra à l'enseignement technique de réaliser son ouvre sans tâtonnement et en toute force.

Quelques notes inédites, maintenant, sur les activités de la Commission d'apprentissage des métiers du bâtiment de Montréal. I.a Loi d'aide à l'apprentissage autorise les Commission d'apprentissage à donner des cours professionnels. Autant que possible une Commission doit utiliser les services d'enseignement technique mis à la disposition de l'industrie ou des métiers. Dans le bâtiment, les initiatives de l'Etat ont été plutôt rares, si bien que l'apprentissage des ouvriers devait forcément

(Suite à la page 6)

Abonnement: $\$ 1.50$ la sêrie de 10 numéros 